\title{
Research Trends and Challenges for Network-Based ICT Systems
}

\author{
Homero Toral-Cruz ${ }^{1}$ - Albena D. Mihovska ${ }^{2}$ • \\ Miroslav Voznak $^{3} \cdot$ Kamaljit I. Lakhtaria $^{4}$. \\ Robert Bestak ${ }^{5}$
}

Published online: 17 May 2015

(C) Springer Science+Business Media New York 2015

Welcome to this special issue of Wireless Personal Communications on Research Trends and Challenges for Network-based ICT Systems. This special issue provide an in-depth research study of the latest trends and challenges for network-based ICT systems.

The convergence of information and communication technologies (ICT), have resulted into significant changes for our network infrastructures. We have witnessed an evolution from a small number of interconnected computer networks to a global socio-technical infrastructure, where people communicate and interact spontaneously in cyberspace and

Homero Toral-Cruz

htoral@uqroo.edu.mx; homerotoralcruz@gmail.com

Albena D. Mihovska

albena@es.aau.dk

Miroslav Voznak

miroslav.voznak@vsb.cz

Kamaljit I. Lakhtaria

kamaljit.ilakhtaria@gmail.com

Robert Bestak

robert.bestak@fel.cvut.cz

1 Department of Sciences and Engineering, University of Quintana Roo, Boulevard Bahía s/n Esq. Ignacio Comonfort, Col. del Bosque, 77019 Chetumal, Quintana Roo, Mexico

2 Center for TeleInfrastruktur, Aalborg University, Fredrik BajersVej 7A, Room C1-113, P.O. Box 159, 9220 Aalborg, Denmark

3 Department of Telecommunication, VSB-Technical University of Ostrava, 17. listopadu 15/2172, 70833 Ostrava, Czech Republic

4 School of Information Technology, Auro University, Earth Space, Opposite ONGC, Hazira Road, Surat 394510, Gujarat, India

5 Department of Telecommunications, Czech Technical University in Prague, Technicka 2, 16627 Prague 6, Czech Republic 
with the cyber physical world, create content and share knowledge over a network of heterogeneous networks. The rapid growth and the emergence of new architectures and a myriad of novel "smart" applications and services. The above-mentioned changes have motivated the development of novel protocols, algorithms, models, tools, techniques and methodologies in order to achieve the desired cooperation and design of network-based ICT architectures for the delivery of smart, secure and robust applications.

This special issue is composed by fifteen high quality original papers covering theoretical and practical aspects on this emerging topic in order to provide novel ideas and directions for the relevant scientific industrial and academic community. We have selected a set of best papers from: (a) KTTO 2014 Conference (Knowledge in Telecommunication Technologies and Optics); (b) three Special Sessions of NAEC 2014 Conference (Networking and Electronic Commerce Research): Special Session on Networks and Communication Systems (NCS 2014), Special Session on Wireless Networking (WN 2014) and Special Session on IP Networks and Protocols (IPNP 2014); and (c) the academic community around the globe (for researchers who could not participate in these conferences). All conference papers have been extended and reviewed again by three independent reviewers.

The special issue starts with the paper entitled "Analysis of slotted-access-based channel access control protocol for LTE-advanced networks" by Wei et al. [1] where an analytical model to investigate the performance of the SCACP for LTE-Advanced (LTEA) networks is proposed. An access cycle decision algorithm is then presented to set the optimal access cycle of SCACP to maximize the utilization of RACHs subject to a given quality of service (QoS) constraint of human traffic. The authors show by means of numerical results the accuracy of the proposed analytical model and the effectiveness of the access cycle decision algorithm.

The second paper entitled "Navigation data-assisted opportunistic spectrum scheduling for network-based UAV systems: A parallel restless bandits formulation” by Si et al. [2] proposes a navigation data-assisted optimal opportunistic spectrum access scheme for wireless communications in heterogeneous UAV networks, to achieve maximized longterm data rate by flexibly scheduling the spectrum subbands. Furthermore, to formulate the optimal spectrum scheduling problem, the authors develop a parallel restless bandits model, which can reduce the NP-hard optimization problem to simply selecting the link with the minimum index in the online stage. Extensive simulation results are also presented to demonstrate the significant performance improvement of the proposed scheme compared to the existing one.

The third paper entitled "Cooperative context data acquisition and dissemination for situation identification in vehicular communication networks" by Kurmis et al. [3] presents a cooperative context data acquisition and dissemination model for situation identification in vehicular communication networks. The authors claim that their solution is different from others as it uses additional virtual context information source. The proposed decision support system decides if the message should be transmitted to other vehicles, sent to the cloud, saved locally or dismissed. The simulation results show the promising context exchange rate between vehicles and huge saving on channel utilization.

The fourth paper entitled "Voice messaging system as a form of distribution of an urgent information" by Rezac et al. [4] discusses a communication system intended to warn people in danger. This system aims to distribute a voice message with pre-recorded content to inform participants about the target events in their area using Voice over IP technology. Transmission of information via voice messages has, compared with other ways of message distribution, the advantage that the target user is forced to pick up the call 
and listen to the message. Another advantage is more accurate addressing of target groups of subscribers and, last but not least, it is possible to ensure the re-delivery of messages to users who did not receive a call.

The fifth paper entitled "Building customer trust in cloud computing with an ICTenabled global regulatory body" by Pathan and Mohammed [5] suggests a different concept of ensuring trust in cloud services by using the power of ICT. The basic idea lies in the fact that there should be a global standardization authority which would certify trusted cloud providers which in turn would earn customer trust. Novelty in this concept is mainly in its operational details presented in the paper. The authors analyze various aspects of this proposed model from the policy making issues alongside slightly addressing technical issues.

The sixth paper entitled "On modeling the psychology of wireless node interactions in the context of internet of things" by Kasabova et al. [6] proposes and evaluates a model for the psychology of the wireless node interactions in the context of IoT and the selection of a partner for the most reliable communication, depending on the needs of the service required. The key functions of the proposed model are the Influence and the Behavior functions. Additionally parameters, which enable the incorporation of different kinds of expectations, reliability levels and service types, are defined.

The seventh paper entitled "Model to increase the number of output states of a random variable using a histogram based PDF" by Klucik et al. [7] is devoted to a pseudo-random number generator that generates numbers according to a known probability density function. Authors proposed model generating practically an unlimited set of possible output states of the random variables. This model was applied on a packet generator that generates IPTV inter-departure times of an MPEG2-TS H.264 VBR based video source.

The eighth paper entitled "A cross layer communication protocol with transmission power adjustment for energy saving in multi-hop WSNs" by Boubiche et al. [8] deals with a new cross-layer design based on three-ware cross-layer architecture which exploits an inversed interaction between network, link and physical layers. The authors propose a Cross Layer and Hybrid Energy Efficiency Protocol (named CL-HEEP) with transmission power adjustment to preserve the energy reserves in Multi-hop WSNs. The main idea is the use of routing information to dynamically adjust the transceiver transmission power and to establish cross-layer duty-cycling. An additional wake-up radio is also implied by CLHEEP to preserve the energy while avoiding compulsory wake-up, idle listening, interference, and collision problems. The simulation reveals that CL-HEEP is energy-efficient and able to achieve significant performance improvement compared to other cross-layer protocols.

The ninth paper entitled "Ant colony optimization based orthogonal directional proactive-reactive routing protocol for wireless sensor networks" by Jain and Reddy [9] proposes an routing protocol named as OD-PPRP which does not require global location information, has low control overhead, is scalable, lightweight and energy aware. The proposed protocol has the characteristics of both reactive and proactive routing protocols and utilizes fuzzy logic and Ant Colony Optimization (ACO) to identify energy efficient and optimal paths. The simulation results show in both static and dynamic environment, that OD-PRRP has better performance compared to other routing protocols.

The tenth paper entitled "Centralized IDS based on misuse detection for cluster-based wireless sensors networks" by Hidoussi et al. [10] proposes a new centralized intrusion detection system to detect selective forwarding and black hole attacks in cluster-based wireless sensors networks. The main idea is the use of a centralized detection approach, where the base station decides on potential intrusions based on control packets sent from 
the cluster heads. The proposed intrusion detection technique is simple and energy efficient, it is thus suitable for sensor nodes with resource constrained. The simulation results confirm the expected performance of the proposed IDS in terms of security and energy efficiency.

The eleventh paper entitled "An efficient remote user authentication with key agreement scheme using elliptic curve cryptography" by Huang et al. [11] proposes an authentication with key agreement scheme using elliptic curve cryptography, which can withstand offline password guessing attacks and impersonation attacks. After the function and efficiency comparison with other schemes, the authors show that their scheme is much more secure and practical as the secure universal access control mechanism.

The twelfth paper entitled "A dynamic-identity based multimedia server client authentication scheme for tele-care multimedia medical information system" by Deebak et al. [12] proposes a dynamic-identity based multimedia server client authentication scheme to resolve the major security threats. Authors proved that the proposed scheme is secure and efficient in comparison to the most used authentication schemes.

The thirteenth paper entitled "A new dynamic id based user authentication scheme using mobile device: cryptanalysis, the principles and design" by Li et al. [13] analyzes the security and design flaws of a recently proposed dynamic ID authentication and key agreement scheme by Lin. The authors propose some principles which should be followed in the design of the user authentication schemes and they designed a new dynamic IDbased user authentication scheme using mobile device.

The fourteenth paper entitled "P2PM-pay: person to person mobile payment scheme controlled by expiration date" by Martínez-Peláez et al. [14] proposes a P2PM-pay scheme which provides two key points. The first key point is related with the mobile cash which is controlled by the expiration date. The other key point is related with the authentication process among participants by means of WTLS protocol and a wireless public key infrastructure with an efficient certificate path validation. The proposed scheme is secure against well-known attacks and efficient in terms of processing time.

Finally, the fifteenth paper entitled "PSP CO2: an efficient hardware architecture for AES algorithm for high throughput" by Karthigaikumar et al. [15] presents a FPGA implementation of high throughput and reduced area architecture for AES encryption and decryption algorithm for wireless communication. In the proposed architecture, the throughput is increased by using Parallel Sub-Pipeline (PSP) architecture and techniques like Composite Field Arithmetic (CFA), on the fly key expansion and order change are combined in order to reduce the AES algorithm implementation area.

This fine selection of papers has been achieved through a fruitful collaboration. We would like to thank the Editor-in-chief (Professor Ramjee Prasad) and Springer's senior editorial assistants (Gabriella Anderson and Meertinus Faber) for their support and help in realizing this special issue. We also would like to give our sincere thanks to all the authors for their contributions and the reviewers for contributing with their expert comments to improve selected papers. Special thanks to the organizers of KTTO (especially to Prof. Miroslav Voznak) and NAEC (especially to Prof. Bezalel B. Gavish and Prof. Michael R. Bartolacci) conferences for gathering quality papers for this special issue. We hope you will enjoy reading this special issue.

Coordinating Guest Editor: Homero Toral-Cruz

Guest Editors: Albena D. Mihovska, Miroslav Voznak, Kamaljit I. Lakhtaria, Robert Bestak 


\section{References}

1. Wei, C.-H., Cheng, R.-G., \& Lin, Y.-S. (2015). Analysis of slotted-access-based channel access control protocol for LTE-advanced networks. Wireless Personal Communications. doi:10.1007/s11277-0152725-3.

2. Si, P., Richard Yu, F., Yang, R., \& Zhang, Y. (2015). Navigation data-assisted opportunistic spectrum scheduling for network-based UAV systems: A parallel restless bandits formulation. Wireless Personal Communications. doi:10.1007/s11277-015-2726-2.

3. Kurmis, M., Andziulis, A., Dzemydiene, D., Jakovlev, S., Voznak, M., \& Gricius, G. (2015). Cooperative context data acquisition and dissemination for situation identification in vehicular communication networks. Wireless Personal Communications. doi:10.1007/s11277-015-2727-1.

4. Rezac, F., Voznak, M., \& Tomala, K. (2015). Voice messaging system as a form of distribution of an urgent information. Wireless Personal Communications. doi:10.1007/s11277-015-2728-0.

5. Pathan, A. S. K., \& Mohammed, M. M. Z. E. (2015). Building Customer trust in cloud computing with an ICT-enabled global regulatory body. Wireless Personal Communications. doi:10.1007/s11277-0152729-z.

6. Kasabova, S., Gechev, M., Vasilev, V., Mihovska, A., Poulkov, V. (2015). On modeling the psychology of wireless node interactions in the context of internet of things. Wireless Personal Communications. doi:10.1007/s11277-015-2730-6.

7. Klucik, S., Chromy, E., \& Baronak, I. (2015). Model to increase the number of output states of a random variable using a histogram based PDF. Wireless Personal Communications. doi:10.1007/s11277-0152731-5.

8. Boubiche, D. E., Bilami, A., Boubiche, S., \& Hidoussi, F. (2015). A cross layer communication protocol with transmission power adjustment for energy saving in multi-hop WSNs. Wireless Personal Communications. doi:10.1007/s11277-015-2732-4.

9. Jain, A., \& Ramana Reddy, B. V. (2015). Ant colony optimization based orthogonal directional proactive-reactive routing protocol for wireless sensor networks. Wireless Personal Communications. doi:10.1007/s11277-015-2733-3.

10. Hidoussi, F., Toral-Cruz, H., Boubiche, D. E., Lakhtaria, K., Mihovska, A., \& Voznak, M. (2015). Centralized IDS based on misuse detection for cluster-based wireless sensors networks. Wireless Personal Communications. doi:10.1007/s11277-015-2734-2.

11. Huang, B., Khan, M. K., Wu, L., Bin Muhaya, F. T., \& He, D. (2015). An efficient remote user authentication with key agreement scheme using elliptic curve cryptography. Wireless Personal Communications. doi:10.1007/s11277-015-2735-1.

12. David, D. B., Rajappa, M., Karupuswamy, T., \& Iyer, S. P. (2015). A dynamic-identity based multimedia server client authentication scheme for tele-care multimedia medical information system. Wireless Personal Communications. doi:10.1007/s11277-015-2736-0.

13. Li, X., Liao, J., Kumari, S., Liang, W., Wu, F., \& Khan, M. K. (2015). A new dynamic id based user authentication scheme using mobile device: Cryptanalysis, the principles and design. Wireless Personal Communications. doi:10.1007/s11277-015-2737-z.

14. Martínez-Peláez, R., Toral-Cruz, H., Ruiz, J., \& Velarde-Alvarado, P. (2015). P2PM-pay: Person to person mobile payment scheme controlled by expiration date. Wireless Personal Communications. doi:10.1007/s11277-015-2738-y.

15. Karthigaikumar, P., Anitha Christy, N., \& Siva Mangai, N. M. (2015). PSP CO2: An efficient hardware architecture for AES algorithm for high throughput. Wireless Personal Communications. doi:10.1007/ s11277-015-2739-x. 

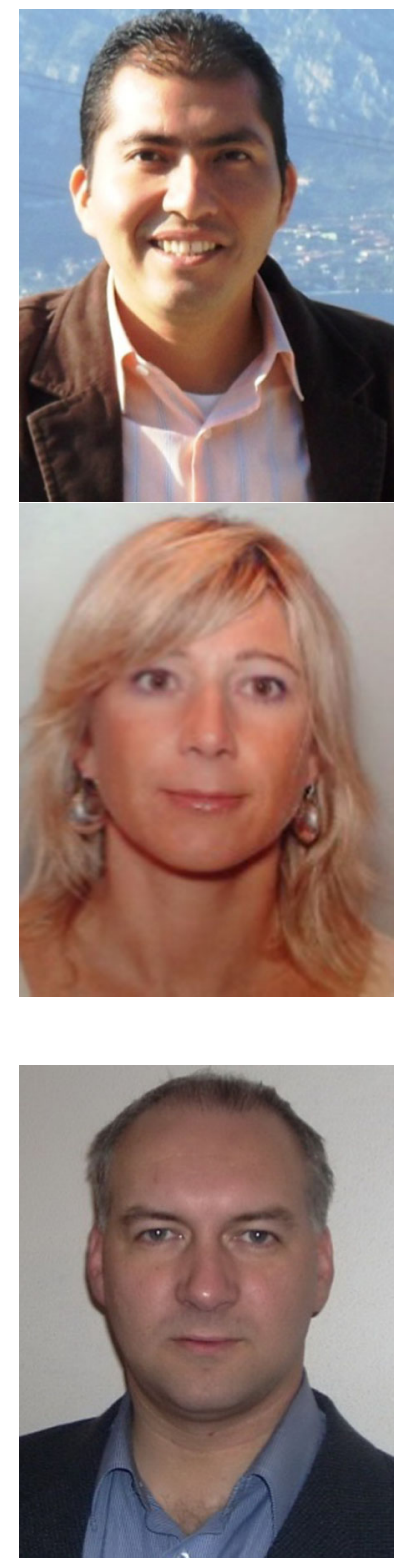

Homero Toral-Cruz received Ph.D. and M.S. degrees in Electrical Engineering, Telecommunication option from Center for Research and Advanced Studies of the National Polytechnic Institute (CINVESTAV), Jalisco, Mexico, in 2006 and 2010, respectively. He received the B.Sc. degree in Electronic Engineering from "Instituto Tecnológico de la Laguna", Coahuila, Mexico in 2002. He is currently an Assistant Professor at Sciences and Engineering department in University of Quintana Roo, Mexico. His research interest includes VoIP technologies, QoS and network measurements, convergent networks, Internet technologies, IP traffic modeling, network performance evaluation, network security and WSN. He has served as Guest Editor of some international journals and TPC member of several international conferences and workshops. He has been awarded a national recognition as a researcher (SNI level C) by CONACYT and has been elected as member of the Mexican Academy of Sciences (AMC).

Albena D. Mihovska has a Ph.D. degree in mobile communications from Aalborg University, Aalborg, Denmark, where she is currently an Associate Professor and Head of Standardisation at the Center for TeleInfrastruktur (CTIF), Aalborg University. Currently, she is involved with research related to innovative research concepts for $5 \mathrm{G}$ communication systems, the design and implementation of eHealth services (EU project eWALL) and to optimizing and supporting reliable and high performance intensive data rate communications as required by the Internet of Things.

Miroslav Voznak (born in 1971) is an Associate professor with Department of Telecommunications, VSB-Technical University of Ostrava. He received his Ph.D. degree in telecommunications, dissertation thesis "Voice traffic optimization with regard to speech quality in network with VoIP technology" from the VSB-Technical University of Ostrava in 2002 and he was appointed as an associate professor in 2009. His professional knowledge covers generally Information and Communication technology, in his research, he deals with wireless networks, Voice over IP, security and optimization problems. He is a senior resarcher in IT4Innovations, Czech National Centre of Excellence in Ostrava and recently he was member of two 7FP EU projects under Grant Agreement No. 218086 (2009-2014) conducted by AGH Cracow and No. 238875 (2009-2013) conducted by GÉANT, Cambridge. He is author or co-author more than one hundred results indexed in SciVerseSCOPUS with more than 200 citations and his h-index $=9$. 


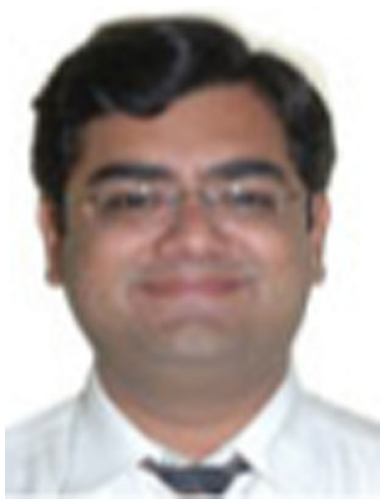

Kamaljit I. Lakhtaria is working as Assistant Professor in School of Information Technology, Auro University. Previous he worked in Sir Padampat Singhaniya University-Udaipur. He obtained Ph.D. in Computer Science in July 2010. His area of research is "Next Generation Networking Service Prototyping and Modeling". He holds an edge in Next Generation Network, Web Services, Mobile Ad Hoc Networks, Network Security and Cryptography. He is author of 9 reference Books in the area of Computer Science. He published 2 chapters in International Editorial Volumes. He presented 30 research papers in International Conferences. His papers are published in the proceedings of IEEE, Springer and Elsevier. He presented 33 research papers in National Conference. He published 35 research papers in referred International Journals and 6 research papers in National Journals. Dr. Kamaljit I. Lakhtaria is supervisor of Ph.D. and M.Phil. research in four Universities. His student complete Ph.D. under his guidance and more two students have submitted their thesis. He has been invited by Anna University, Bharathiar University, Saurashtra University, Pacific University as subject expert for thesis evaluation as external referee. He is life time member ISTE, IAENG and many research groups. He hold the post of Editor, Associate Editor in many International Research Journal. He is program committee member of many International conferences. He is reviewer in IEEE WSN, Inderscience and Elsevier Journals.

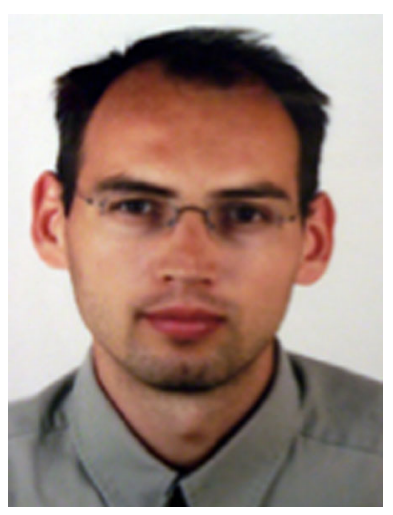

Robert Bestak obtained the Ph.D. degree in Computer Science from ENST Paris, France (2003) and MSc degree in Telecommunications from Czech Technical University in Prague, CTU, (1999). Since 2004, he has been an Assistant Professor at Department of Telecommunication Engineering, Faculty of Electrical Engineering, and CTU. His main research interests include $5 \mathrm{G}$ networks, big data in mobile network, and spectrum management. He is the Czech representative in the IFIP TC6 working group. He has served as Steering and Technical Program Committees member for numerous IEEE/IFIP international conferences (Networking, WMNC, NGMAST, etc.). He participated in several national, EU, and third party founded research projects. 